\title{
USO POTENCIAL DE COMPONENTES DEL VENENO DE SERPIENTE EN EL TRATAMIENTO DEL CÁNCER
}

\author{
Dan Vivas ${ }^{1, a}, R^{2} s_{i} I_{n g a}^{1,2, a}$, Armando Yarlequé ${ }^{1, b}$.
}

RESUMEN

El desarrollo del cáncer es posible en la medida que las células tumorales proliferen, se dispersen e invadan otros tejidos del cuerpo. Las integrinas son una familia de receptores heterodiméricos de superficie celular que cumplen un papel crucial en el desarrollo de la angiogénesis, crecimiento y metástasis de un tumor señalándolas como un atractivo blanco terapéutico. Los venenos de serpientes contienen péptidos de bajo peso molecular conocidos como desintegrinas, las que se unen con una alta afinidad a las integrinas e inhiben su accionar en un proceso cancerígeno. En el siguiente articulo revisamos los resultados de investigaciones, tanto in vitro como in vivo, que han mostrado resultados promisorios, por lo cual el uso de las desintegrinas podrían constituir una alternativa promisoria para el tratamiento de diversas neoplasias.

Palabras clave: Cáncer; Venenos de serpiente; Desintegrinas; Tratamiento (fuente: DeCS BIREME).

\section{POTENTIAL USE OF SNAKE VENOM COMPONENTS IN CANCER TREATMENT}

\begin{abstract}
Cancer can develop to the extent tumor cells grow, divide and grow into other body tissues. Integrins are a family of cell-surface heterodimeric receptors that play an important role in the development of tumor angiogenesis, growth and metastasis, thus being recognized as an attractive therapeutic target. Snake venom contains low-molecular weight peptides known as "disintegrins" that bind to integrins with high affinity, and prevent their action in cancer. In the next article, we go over the results of investigations, both in vitro and in vivo, which have shown promising results, thus revealing that the use of disintegrins could be a promising alternative for the treatment of different neoplasias.
\end{abstract}

Key words: Neoplasms; Snake venoms; Disintegrins; Treatment (source: MeSH NLM).

\section{INTRODUCCIÓN}

Las células cancerígenas reestructuran vías completas de señalización metabólica que permiten su proliferación ilimitada; los requerimientos para su superviviencia, migración e invasividad dependen de la participación de variadas moléculas (factores de crecimiento, integrinas), y de la interacción con otras células y la matriz extracelular (MEC). Las terapias contra el cáncer se centran en la búsqueda de moléculas antogonistas que impidan las interacciones de las células neoplásicas con su entorno (bloqueando su proliferación, su migración, e induciendo su apoptosis) ${ }^{(1,2)}$. El presente artículo da a conocer el papel que poseen las desintegrinas ofídicas como potenciales inhibidores de la angiogénesis y la metástasis.

\section{LA ANGIOGÉNESIS Y LA METÁSTASIS}

La angiogénesis es un proceso esencial altamente regulado que normalmente se da en eventos de desarrollo embrionario, crecimiento y reparación de heridas. Sin embargo, en un desarrollo tumoral, este proceso se torna continuo permitiendo el suministro de oxígeno y nutrientes ${ }^{(1)}$.

Un tumor induce la angiogénesis cuando sus células, en un estado de hipoxia, producen una molécula conocida el factor alfa hipoxia inducible (HIF- $\alpha$ ) que estimula la producción del factor de crecimiento de endotelio vascular A (VEGF-A), activador angiogénico mejor conocido a la fecha ${ }^{(2,3)}$. Este activador se une

\footnotetext{
Laboratorio de Biología Molecular, Facultad de Ciencias Biológicas, Universidad Nacional Mayor de San Marcos. Lima, Perú.

Laboratorio de Reactivos de Diagnóstico, Centro Nacional de Productos Biológicos, Instituto Nacional de Salud. Lima, Perú.

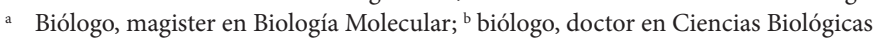

Recibido: 26-04-12 Aprobado: 08-08-12

Citar como: Vivas D, Inga R, Yarlequé A. Uso potencial de componentes del veneno de serpiente en el tratamiento del cáncer. Rev Peru Med Exp Salud Publica. 2012;29(3):396-401.
} 
preferentemente a un tipo de receptor tirosina quinasa (VEGFR-2), ubicado en las células endoteliales (4) y conlleva a una respuesta angiogénica que consiste en la formación de nuevos brotes vasculares y la eventual formación de vasos sanguíneos hacia el tumor ${ }^{(5,6)}$, cuya estabilidad requiere de otros factores de crecimiento pro angiogénicos tales como el factor de crecimiento derivados de plaquetas (PDGF), factor de crecimiento de fibroblastos (FGF) y del factor de crecimiento epidermal (EGF) así como también oxido nítrico, prostaglandinas y factores de transcripción Ets-1 ${ }^{(6,7)}$.

La unión de VEGF-A al receptor VEGFR-1 está relacionada con los procesos de hematopoyesis y el reclutamiento de monocitos y de otras células derivadas de la medula ósea hacia la neovasculatura que potencian la angiogénesis. El VEGFR-1 también participa en la liberación paracrina de factores de crecimiento de las células endoteliales y en la secreción de metaloproteinasas de la matriz (MMPs), que degradan la membrana basal ${ }^{(3)}$.

Los vasos sanguíneos neoplásicos son anormales en muchos aspectos: tienen grandes dilataciones, muchas extensiones, puentes, divisiones y sus paredes son un mosaico de células endoteliales y tumorales; este mosaicismo permite la entrada de las células tumorales al torrente sanguíneo para su diseminación, fenómeno conocido como metástasis ${ }^{(8)}$.

Para diseminarse, las células tumorales rompen sus lazos con la estructura cohesiva del tejido de origen reduciendo su adhesividad por la pérdida de proteínas de anclaje ${ }^{(9)}$ o como parte de la transformación de células cancerosas de un estado epitelial a un estado de mayor movilidad conocido como transición epitelio mesenquimal ${ }^{(10)}$.
Las interacciones de las células tumorales con las células endoteliales desempeñan un papel crítico en la diseminación vascular de los tumores, tanto en la intravasación hacia el espacio vascular, como en la extravasación hacia los tejidos (11). El desarrollo de este proceso se da por la participación de grupos de proteínas como las tetraspaninas ${ }^{(12)}$, las proteínas ADAM (A Disintegrin-like and Metalloproteinasa) ${ }^{(6)}$ y un tercer grupo que participa en la metástasis una vez iniciada, las integrinas, que median las interacciones de las células tumorales con la matriz extracelular (MEC) ${ }^{(13)}$.

\section{INTEGRINAS}

Lasintegrinas son una familia de receptores heterodiméricos de superficie celular que cumplen un rol importante en la adherencia celular a la MEC. Estas glicoproteínas, constituidos por una subunidad $\alpha$ y una $\beta$, transmiten señales tanto del entorno como del interior celular que influyen en la forma, supervivencia, proliferación y migración celular. Esta familia forma por lo menos 24 pares distintos por la combinación de sus 18 subunidades a y 8 subunidades $\beta$ siendo, cada par, específico para un grupo de ligandos únicos. Las integrinas reconocen motivos RGD (aminoácidos Arg-Gly-Asp) presentes en las proteínas de la matriz extracelular, para realizar las interacciones entre el citoesqueleto y la MEC ${ }^{(13)}$.

La expresión y distribución de las integrinas varían entre los tumores malignos y tumores preneoplásicos del mismo tipo ${ }^{(14)}$. En una metástasis las células están constantemente formando y rompiendo contactos de integrinas, requerimiento fundamental para que las células adquieran la tracción necesaria en su movimiento a través de la degradación y remodelación de la MEC por
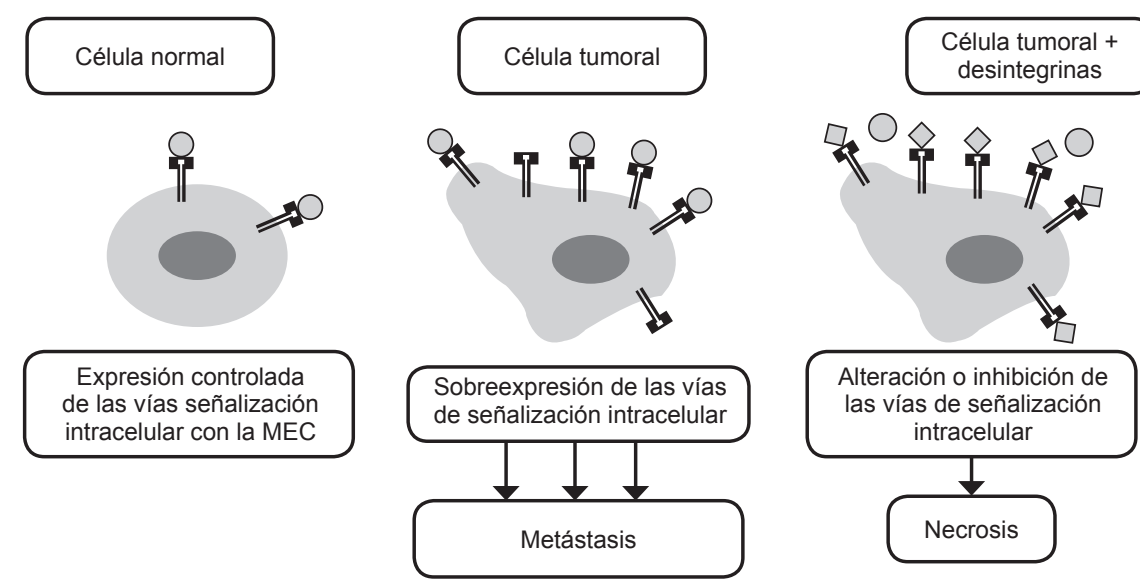

Figura 1. Esquema de la expresión de las integrinas y mecanismo de acción sugerente de las desintegrinas.

MEC: matriz extracelular. 
efecto de las MMPs que actúan sobre las proteínas de la membrana basal; esto a su vez, promueve la activación de varias vías de señalización intracelular (FAK, RHO, CDC, RAC, ERK, PI3K, SHC, etc.) que controlan la reorganización del citoesqueleto, la generación de fuerza y la anulación de la apoptosis ${ }^{(14)}$.

\section{DESINTEGRINAS}

Las desintegrinas son péptidos de bajo peso molecular (4-16 kDa) encontrados, como tales o formando dominios en las metaloproteinasas de la clase P-III, en todos los venenos de serpientes estudiadas hasta la fecha (familias Atractaspididae, Elapidae, Viperidae y Colubridae) ${ }^{(15,16)}$. Estos péptidos forman parte de la gama de moléculas ofídicas que la biomedicina estudia en los últimos años, como las neurotoxinas, dendrotoxinas, citotoxinas, miotoxinas, cardiotoxinas, lectinas, enzimas similares a trombina, activadores de protrombina, etc. ${ }^{(17)}$.

La característica principal de las desintegrinas es su habilidad para interactuar y alterar la actividad de las integrinas ${ }^{(15)}$. El estudio de las desintegrinas en la inhibición de la agregación plaquetaria dio a conocer que estas moléculas poseen el motivo RGD o motivos similares, que permiten su unión de una manera dosis dependiente a las integrinas de la superficie celular ${ }^{(16)}$.

\section{CLASIFICACIÓN DE LAS DESINTEGRINAS}

Funcionalmente, las desintegrinas se clasifican en tres grupos de acuerdo con su selectividad a la integrina y a la presencia de motivos específicos. Estos grupos son $(A)$ desintegrinas que interactúan con las integrinas motivo RGD-dependientes (B) desintegrinas de unión a integrinas de leucocitos y $(C)$ las desintegrinas de unión a integrinas $\alpha 1 \beta 1$. El primer grupo incluye a la mayoría de desintegrinas monoméricas con motivos RGD y las desintegrinas con motivos KGD, MVD, MGD y WGD. El segundo grupo presentan el motivo MLD, que interactúan con las integrinas $\alpha 4 \beta 1, \alpha 4 \beta 7$ y $\alpha 9 \beta 1$. El tercer grupo, lo integran desintegrinas con el motivo KTS que son potentes y selectivos inhibidores de las integrinas $\alpha 1 \beta 1$ (receptores específicos del colágeno tipo IV) ${ }^{(15)}$.

Estructuralmente, son clasificadas en cortas (41-51 aminoácidos [aa] y 4 puentes disulfuros [S-S]); medianas (70 aa y $6 \mathrm{~S}-\mathrm{S}$ ); largas (84 aa y $7 \mathrm{~S}-\mathrm{S}$ ) y diméricas (con cerca de 67 aa y de 2 a 4 S-S intracatenarios). También se considera el grupo de disintegrin-like domain (dominio similar a desintegrina) que se encuentran en las metaloproteasas de la clase P-III; este grupo de 100 aa y 8 S-S contienen un extremo C-terminal de 110 residuos de cisteínas con 6 S-S ${ }^{(16)}$.

\section{DESINTEGRINAS OFIDICAS Y SUS POTENCIALES PROPIEDADES ANTICANCERÍGENAS}

Estudios iniciales descubrieron la capacidad de las desintegrinas para inhibir la agregación plaquetaria. Luego se observó que prevenían la adhesión de líneas celulares tumorales a los componentes de la matriz extracelular ${ }^{(6)}$. Sheu et al. reportaron por primera vez la actividad antiangiogénica de una desintegrina, triflavin (de Trimeresurus flavoviridis) que inhibía la angiogénesis sobre las células endoteliales de la vena umbilical humana (HUVEC) mediante la unión a la integrina av $\beta 3$ de una forma más efectiva que un anticuerpo específico ${ }^{(18)}$. Una serie de investigaciones en diferentes venenos ofídicos demostraron que las desintegrinas pueden inhibir, de forma dosis dependiente, el proceso clave para el desarrollo de un cáncer: la angiogénesis.

La desintegrina homodimérica contortrostatin reduce la densidad de la microvasculatura en líneas celulares de cáncer de mama MDA-MB-435, bloqueando su crecimiento. Asimismo inhibe, in vitro, la angiogénesis del glioblastoma multiforme, un tipo de cáncer resistente a la cirugía, radiación y quimioterapia, al unirse fuertemente a las integrinas responsables de la cascada de señalización vía FAK $^{(6,15,19)}$.

La desintegrina monomérica echistatin (de Echis carinatus) impide la formación de capilares en modelos de membrana corionalontoidea (CAM). En las células T24, de carcinoma de vejiga humana, su unión a la integrina $\alpha \mathrm{V} \beta 3$ inhibe la adhesión a láminas de fibronectina y también desprende la fibronectina de células GD25 de ratón induciendo su apoptosis por medio de la disminución de los eventos de fosforilación en la vía FAK ${ }^{(15,20)}$

El saxatilin (de Glodyus saxatilis) inhibe la angiogénesis y la proliferación celular de melanomas en ratones e inhibe la proliferación de líneas celulares de cáncer ovárico humano inducida por TNF- $\alpha$, evidenciando un posible mecanismo de inhibición de la angiogénesis al bloquear la acción de quimoquinas. Inhibe también la liberación del PDGF-AB impidiendo la adhesión plaquetaria activadas por colágeno y bloquea el efecto angiogénico de líquido derivado de plaquetas que promueve la migración e invasión en HUVEC (21).

Las desintegrinas obtustatin y lebestatin, con motivos KTS, poseen la habilidad de bloquear los procesos centrales de la angiogénesis y la progresión tumoral, actuando como antagonistas de las integrinas $\alpha 1 \beta 1$ en estudios in vivo (usando modelos murinos y CAM). Específicamente, obustatin inhibe el desarrollo de nueva 
vasculatura en modelos CAM, el desarrollo de carcinoma pulmonar de Lewis, así como también la adhesión de los eosinófilos al colágeno tipo IV. El lebestatin, impide la angiogénesis inducida por PDGF y VEGF evitando la adhesión y migración de CHO- a1, PC12 y HUVEC al colágeno tipo I y IV ${ }^{(15,22)}$.

La desintegrina heterodimérica VLO5, con el motivo MLD, bloquea la proliferación y la angiogénesis de las células endoteliales de la microvasculatura dérmica humana (dHMVEC) expresantes de la integrina $\alpha 9 \beta 1$, al igual que las células del cerebro (bHMVEC) que no expresa $\alpha 9 \beta 1^{(15)}$.

El accutin (de Agkistrodon acutus), inhibe la adhesión y migración de HUVEC, exhibiendo además efectos antiangiogénicos tanto in vitro como in vivo (sobre modelos CAM) ${ }^{(15)}$. El salmosin (de Agkistrondon halys), actúa reduciendo la metástasis en el cáncer pulmonar, en líneas celulares SK-Mel 2, previniendo el crecimiento de melanomas al unirse a la integrina $\alpha \mathrm{V} \beta 3{ }^{(15,23)}$.

En células cancerígenas de pulmón, la desintegrina DisBa01 (de Bothrops alternatus) inhibe la angiogénesis inducida por el FGF mediante su unión a la integrina aVß3. DisBa01 inhibe la adhesión de la línea celular 1 de endotelio microvascular humano (HMEC-1) y de la línea celular de melanoma murino B16F10 ${ }^{(24)}$.

El bitistatin (de Bitis arietans) impide la proliferación de carcinomas mamarios en ratones al unirse a las integrinas aVß3 y allb $\beta 3$. Trigramin (de Trimeresurus gramineus) inhibe el crecimiento de las células cancerígenas de mama MDA-MB-231 e impide el acceso de células tumorales a la lámina basal. Agkistin, (de Agkistrondon halys) en modelos CAM, muestra un marcado efecto antiangiogénesis sin afectar los vasos sanguíneos preexistentes. Asimismo, impide la formación del tubo sanguíneo en un cultivo de células endoteliales aórticas hechas en geles de colágeno ${ }^{(25)}$.

También existen otras desintegrinas que muestran propiedades anticancerígenas en el ámbito de crecimiento tumoral (kistrin), angiogénesis (angiostatin $\mathrm{K} 1-3$, endostatin y eristostatin), metástasis (crotatroxin 2); agregación plaquetaria (piscivostatin, ussuristatin, horrdistatin, mojastin y barbourin), adhesión y señalización celular; motilidade interacción conleucocitos (viperistatin, elegantin, VLO4, EO5, rhodostomin, EC3, jarastatin y bitisgabonin) ${ }^{(15)}$.

Por otra parte, las formas recombinantes de desintegrinas muestran interacciones más fuertes que sus formas nativas. La forma recombinante de salmosin (de Agkistrodon halys brevicaudus), expresada en
E.coli, bloqueó la neovascularización en modelos CAM al igual que la proteína nativa, pero además, mostró tener efecto antiproliferativo in vitro y antimetástico in vivo, específicamente contra una línea celular de melanoma, medido por un ensayo de colonización de tumor pulmonar en roedores. Semejantes efectos dieron: r-bothrostatin, albolatin, agkistin-s, jerdostatin, rodostomin, y barbourin ${ }^{(15)}$.

\section{LA COMBINACIÓN DE DESINTEGRINAS CON LIPOSOMAS}

Pocas desintegrinas generan antigenicidad en modelos murinos; el uso de liposomas, vesículas de doble capa de fosfolípidos donde se introduce un determinado compuesto, representa una alternativa idónea para poder manejar este evento ${ }^{26)}$. Kim et al. quienes previamente habían generado exitosamente una versión recombinante de la desintegrina salmosin, desarrollaron una novedosa propuesta para la entrega de la desintegrina en un sistema biológico (tumores B16BL6). El gene salmosin, secuencia de ADN que codifica para la proteína salmolisin, fue encapsulado dentro de liposomas catiónicos y administrado vía subcutánea; los resultados fueron exitosos ya que se inhibió el crecimiento del melanoma y se suprimió la metástasis pulmonar en ratones ${ }^{(27)}$.

De una manera similar, la forma liposomal de contortrostatin mostró una potente actividad antiangiogénica en un ortotópico y xenógrafo modelo de cáncer de mama humano en roedores, inclusive, la forma liposomal fue mucho más efectiva en reducir el volumen de crecimiento tumoral que la forma no liposomal ${ }^{(26)}$.

\section{DESINTEGRINAS EN EL VENENO DE LAS SERPIENTES PERUANAS}

El Perú cuenta con una considerable fauna ofídica venenosa. Las investigaciones realizadas, básicamente en serpientes del género Bothrops y Lachesis, dan luces de la presencia de potenciales agentes terapéuticos. Recientemente, Koholoff et al. dieron a conocer la presencia de desintegrinas en los venenos de las serpientes peruanas Bothrops atrox, B. pictus y B. barnetti, cuyo porcentaje es 3,$2 ; 8,9$ y $5,5 \%$ respectivamente ${ }^{(28)}$. Sin embargo, no se ha reportado aún ninguna caracterización de sus potencialidades anticancerígenas.

\section{CONCLUSIONES Y PERSPECTIVAS}

Actualmente las desintegrinas son consideradas en el tratamiento de la adipogénesis y su estructura ha servido como molde para la producción de las drogas 
eptifibatide (Integrilin $®$ ) y tirofiban (Aggrastat $®$ ), usadas para la terapia del síndrome de isquemia coronaria aguda y las complicaciones trombóticas ${ }^{(15,16)}$.

Basado en el principio que la adhesión celular mediada por las integrinas permite potencializar la angiogénesis y metástasis; el uso de las desintegrinas constituiría una acción específica en la inhibición de estos dos procesos claves. Es conocido que los tumores logran adquirir resistencia a ciertos tratamientos los cuales ejercen una presión selectiva positiva sobre los tumores cuando las drogas son dirigidas hacia solo un blanco. Las desintegrinas mimetizan el ligando natural de las integrinas, por lo que el desarrollo de una resistencia es poco probable.

La eficacia de las desintegrinas dependerá en gran medida de la estrategia de suministro, y los liposomas muestran ser uno de los criterios más óptimos para evitar un proceso de inmunidad. Se ha demostrado que la especificidad de las desintegrinas puede ser incrementada cuando se la encapsula en partículas virales. El empleo de las formas recombinantes de las desintegrinas es otra alternativa.

Finalmente, el uso combinado de las desintegrinas con las drogas que actualmente se usan en la terapia anticancerígena, darían nuevas perspectivas en la ardua lucha contra el cáncer por lo que el estudio de la presencia de estos péptidos en la ponzoña de especies peruanas se hacen pertinentes y necesarias.

Contribuciones de autoría: DV y RI participaron en la búsqueda de información y la primera redacción del artículo, AY y DV participaron en la revisión crítica y la segunda redacción del artículo RI y AY dieron las sugerencias editoriales finales. Todos los autores aprobaron la versión final del artículo.

Fuentes de financiamiento: Consejo Superior de Investigación de la Universidad Nacional Mayor de San Marcos.

Conflictos de interés: los autores declaran no tener conflictos de interés en la publicación de este artículo.

\section{REFERENCIAS BIBLIOGRÁFICAS}

1. Carmeliet P. Angiogenesis in life, desease and medicine. Nature. 2005;438(7070):932-6.

2. Ferrara N, Gerber HP, LeCouter J. The biology of VEGF and its receptors. Nature Med. 2003;9(6):669-76.

3. Ferrara N, Kerbel RS. Angiogenesis as a therapeutic target. Nature. 2005;438(7070):967-74.

4. Ferrara N. Vascular endothelial growth factor: basic science and clinical progress. Endocr. Rev. 2004;25(4): 581-611.

5. McColl BK, Stacker SA, Achen MG. Molecular regulation of the VEGF -- family inducers of angiogenesis and lymph angiogenesis. APMIS 2004;112(7-8):463-80.

6. Swenson S, Ramu S, Markland FS. Antiangiogenesis and RGD-containing snake venom disintegrins. Curr Pharm Des. 2007;13(28):2860-71.

7. Fraga A, Ribeiro R, Medeiros R. Hipoxia tumoral. Papel del factor inducible por hipoxia. Actas Urol Esp. 2009;33(9):941-51.

8. Maniotis AJ, Folberg R, Hess A, Seftor EA, Gardner LM, Pe'er J, et al. Vascular channel formation by human melanoma cells in vivo and in vitro: vasculogenic mimicry. Am J Pathol. 1999;155(3):739-52.
9. Chiang AC, Massagué J. Molecular basis of metastasis. N Engl J Med. 2008;359(26):2814-23.

10. Guarino M, Rubino B, Ballabio G. The role of epithelial-mesenchymal transition in cancer pathology. Pathology. 2007;39(3):305-18.

11. Kawaguchi T. Cancer metastasis: characterization and identification of the behavior of metastatic tumor cells and the cell adhesion molecules, including carbohydrates. Curr Drug Targets Cardiovasc Haematol Disord. 2005;5(1):39-64.

12. Barreiro O, Sánchez-Madrid F. Bases moleculares de las interacciones leucocito-endotelio durante la respuesta inflamatoria. Rev Esp Cardiol. 2009;62(5):552-62.

13. Nemeth JA, Nakada MT, Trikha M, Lang Z, Gordon MS, Jayson GC, et al. Alpha-v integrins as therapeutic targets in oncology. Cancer Invest. 2007;25(7):632-46.

14. Hood JD, Cheresh DA. Role of integrins in cell invasion and migration. Nat Rev Cancer. 2002;2(2):91-100.

15. McLane MA, Joerger T, Mahmoud A. Disintegrins in health and disease. Front Biosci. 2008;13:6617-37.
16. Calvete JJ, Marcinkiewicz C, Monleón D, Esteve V, Celda B, Juárez $\mathrm{P}$, et al. Snake venom disintegrins: evolution of structure and function. Toxicon. 2005;45(8):1063-74.

17. Koh DC, Armugam A, Jeyaseelan K. Snake venom components and their applications in biomedicine. Cell Mol Life Sci. 2006;63(24):3030-41.

18. Sheu JR, Yen MH, Kan YC, Hung WC, Chang PT, Luk HN. Inhibition of angiogenesis in vitro and in vivo: comparison of the relative activities of triflavin, an Arg-GlyAsp-containing peptide and antialpha(v)beta3 integrin monoclonal antibody. Biochim Biophys Acta; 1997;1336(3):445-54.

19. Minea R, Swenson S, Costa F, Chen TC, Markland FS. Development of a novel recombinant disintegrin, contortrostatin, as an effective antitumor and anti-angiogenic agent. Pathophysiol Haemost Thromb. 2005;34(4-5):177-83.

20. Miltyk W, Surazyński A, Sławomir W, Pałka JA. Combined therapy with disintegrin and melphalan as a new strategy in inhibition of endometrial cancer cell line (Ishikawa) growth. Folia Histochem Cytobiol. 2009; 47(5):S121-5. 
21. Jang YJ, Kim DS, Jeon OH, Kim DS. Saxatilin suppresses tumor-induced angiogenesis by regulating VEGF expression in NCI-H460 human lung cancer cells. J Biochem Mol Biol. 2007;40(3):439-43.

22. Brown MC, Staniszewska I, Del Valle L, Tuszynski GP, Marcinkiewicz C. Angiostatic activity of obtustatin as alphalbeta1 integrin inhibitor in experimental melanoma growth. Int J Cancer. 2008;123(9):2195-203.

23. Chung KH, Kim SH, Han KY, Sohn YD, Chang SI, Baek KH, et al. Inhibitory effect of salmosin, a Korean snake venomderived disintegrin, on the integrin alphav-mediated proliferation of SKMel-2 human melanoma cells. J Pharm Pharmacol. 2003;55(11):1577-82.
24. Ramos OH, Kauskot A, Cominetti MR, Bechyne I, Salla Pontes CL, Chareyre F, et al. A novel alpha(v)beta (3)-blocking disintegrin containing the RGD motive, DisBa-01, inhibits bFGFinduced angiogenesis and melanoma metastasis. Clin Exp Metastasis. 2008;25(1):53-64.

25. Ren A, Wang S, Cai W, Yang G, Zhu Y, WuX,Zhang Y.Agkistin-s, a disintegrin domain, inhibits angiogenesis and induces BAECs apoptosis. J Cell Biochem. 2006;99(6):1517-23.

26. Swenson S, Costa F, Minea R, Sherwin RP, Ernst W, Fujii G, et al. Intravenous liposomal delivery of the snake venom disintegrin contortrostatin limits breast cancer progression. Mol Cancer Ther. 2004;3(4):499-511.
27. Kim SI, Kim KS, Kim HS, Kim DS, Jang Y, Chung $\mathrm{KH}$, et al. Inhibitory effect of the salmosin gene transferred by cationic liposomes on the progression of B16BL6 tumors. Cancer Res. 2003;63(19):6458-62.

28. Kohlhoff M, Borges M, Yarleque A, Cabezas C, Richardson M, Sanchez E. Exploring the proteomes of the venoms of the Peruvian pit-vipers Bothrops atrox, B. barnetti and B. pictus. J Proteomics. 2012;75:2181-95.

Correspondencia: Dan Vivas Ruiz

Dirección: Mz M2 Lt 22 Urb. El Pinar.

Lima 07, Perú.

Teléfono: (51) 994346535

Correoelectrónico:devivasr@hotmail.com

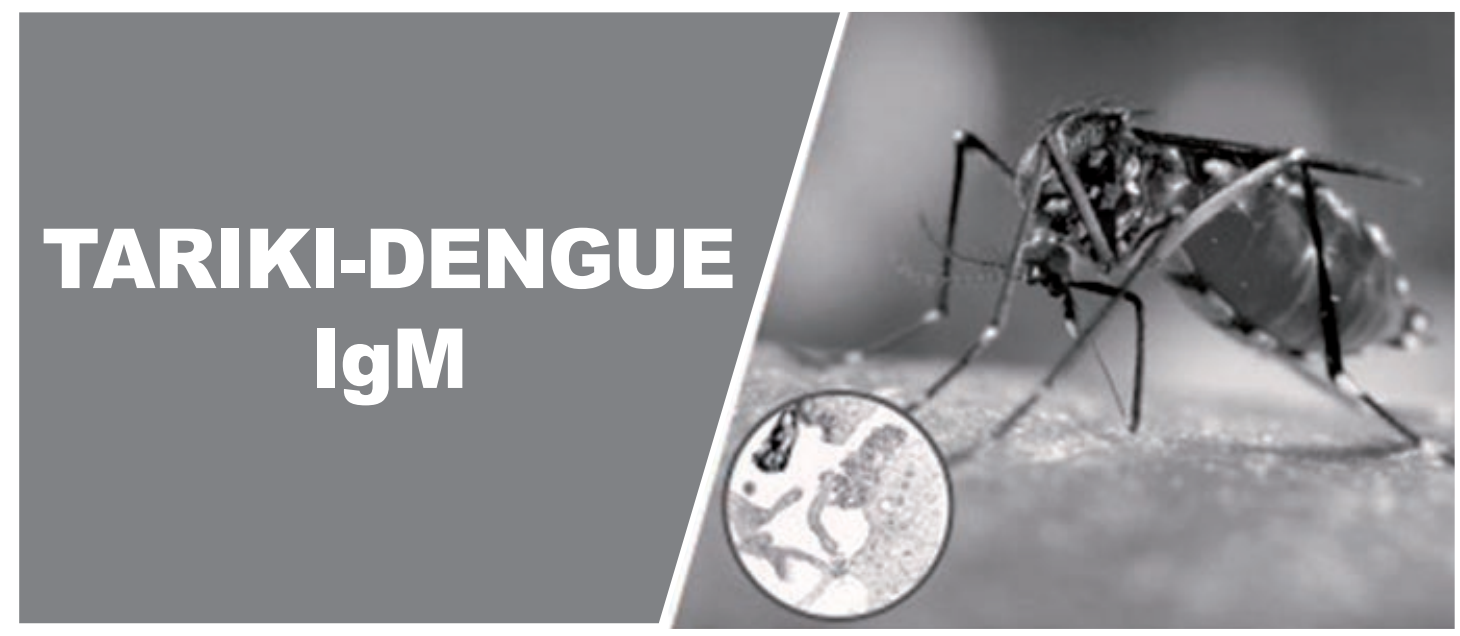

\title{
ELISA de CAPTURA IgM DENGUE
}

\section{TARIKI: disponible en el Perú}

\author{
Kit para la determinación de anticuerpos IgM anti Dengue, \\ desarrollado y producido por el Instituto Nacional de Salud, Perú \\ INS/MINSA
}

